\title{
Models of liver fibrosis: exploring the dynamic nature of inflammation and repair in a solid organ
}

\author{
John P. Iredale
}

Medical Research Council/University of Edinburgh Centre for Inflammation Research, Queen's Medical Research Institute, Edinburgh, United Kingdom.

\begin{abstract}
Models of liver fibrosis, which include cell culture models, explanted and biopsied human material, and experimental animal models, have demonstrated that liver fibrosis is a highly dynamic example of solid organ wound healing. Recent work in human and animal models has shown that liver fibrosis is potentially reversible and, in specific circumstances, demonstrates resolution with a restoration of near normal architecture. This Review highlights the manner in which studies of models of liver fibrosis have contributed to the paradigm of dynamic wound healing in this solid organ.
\end{abstract}

\section{Introduction and clinical relevance}

Liver fibrosis and the end-stage of liver fibrosis, cirrhosis, represent the final common pathway of virtually all chronic liver diseases $(1,2)$. Advanced fibrosis is characterized by an accumulation of ECM rich in fibrillar collagens (predominantly collagen I and collagen III). It results in liver failure and portal hypertension and is associated with an increased risk of liver cancer (2). The wide geographic distribution and high prevalence of insults with the potential to cause liver fibrosis, including chronic viral hepatitis, non-alcoholic steatohepatitis (which is associated with obesity and the metabolic syndrome), parasitemia, inborn errors of metabolism, and toxic damage through alcohol consumption, mean that fibrosis and cirrhosis of the liver remain major causes of morbidity and mortality worldwide (3). In the United Kingdom alone, liver disease is now the fifth most common cause of mortality, and its incidence is rising (3).

Currently the only effective available treatment for cirrhosis of the liver is transplantation. However, shortages of organs, the presence of concurrent disease affecting other tissues in the potential recipient, and recrudescence of the original disease in transplant recipients limit the impact of this treatment and lead to a clear imperative to develop antifibrotic therapies (3-5). Striking increases in our understanding of the pathogenesis of liver fibrosis, with respect to its natural progression and potential evolution, have occurred in the last 20 years. These observations, which include the identification of the main cellular effectors of liver fibrosis, the key cytokines regulating the fibrotic process, and the determinants of ECM turnover, have highlighted a number of potential therapeutic approaches that are likely to be developed in the near future (for detailed review, see ref. 5).

This dramatic change in our understanding of the process of fibrosis has been greatly facilitated by the use of multiple complementary experimental model systems - the subject of this Review. As our understanding of the pathogenesis of liver fibrosis has evolved, it has become clear that the liver provides a very useful generic model of inflammation and repair, demonstrating inter-

Nonstandard abbreviations used: BDL, bile duct ligation; EMT, epithelialmesenchymal transition; HSC, hepatic stellate cell; TIMP, tissue inhibitor of metalloproteinases.

Conflict of interest: J.P. Iredale is employed as a consultant by General Electric Corporation Healthcare to advise on imaging of hepatic fibrosis.

Citation for this article: J. Clin. Invest. 117:539-548 (2007). doi:10.1172/JCI30542. play between the epithelial, inflammatory, myofibroblast, and ECM components of the mammalian wound-healing response (5-7). This Review focuses on two concepts that have recently emerged that emphasize the dynamic nature of liver fibrosis: first, the paradigm that liver myofibroblasts might arise from multiple cell lineages; second, the paradigm that liver fibrosis is a potentially reversible process such that the liver provides a model for inflammation and repair that demonstrates both fibrosis (scarring) and resolution with remodeling and restitution of normal, or near normal, architecture.

\section{Approaches to modeling liver fibrosis}

Models of liver fibrosis can be assigned to three broad groups, each of which has specific advantages and disadvantages. The first group includes cell culture models, in which cultures of highly purified primary cells from normal or experimentally injured livers are isolated and studied in vitro (8-12). This type of model facilitates the detailed study of cell behavior and the effect of specific mediators, but it clearly cannot recapitulate the events that occur in vivo, which result from the complex interplay of resident and incoming cells in a microenvironment. The second group includes human tissues taken at biopsy or following hepatic resection. Studies involving this type of model are essential for validating observations made in tissue culture and animal model systems (13-15). The careful selection of samples and application of modern molecular techniques means that a surprisingly large amount of information can be generated from such tissue. Nevertheless, ethical considerations prevent multiple liver biopsies being taken from patients for research purposes, with the result that the information tends to be limited to "snapshot" data. In addition, the data generated also tends to be representative only of relatively advanced disease. To address and resolve the issues raised by the use of the first two types of model, as well as to describe a potentially dynamic process, a third type of model is used that includes experimental animal models of fibrosis (for detailed review, see refs. 16 and 17). Animal models have the single, large disadvantage that they are not human. However, they allow the serial sampling of tissue in the volume required for detailed studies of cellular and molecular pathogenesis. Additionally, the development of modern molecular tools and genetically modified mice means that mouse models can be applied to mechanistic studies of fibrosis in which individual mediators or cell types are dysregulated. 


\section{Normal liver}

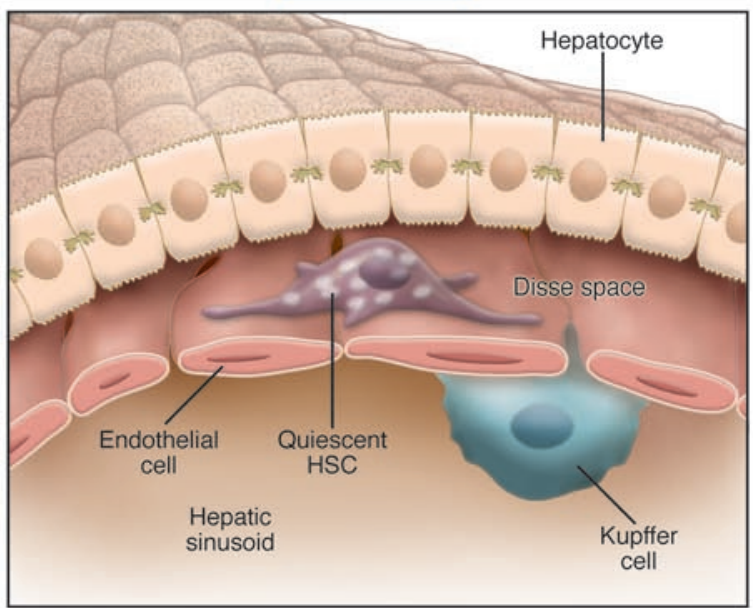

Liver injury

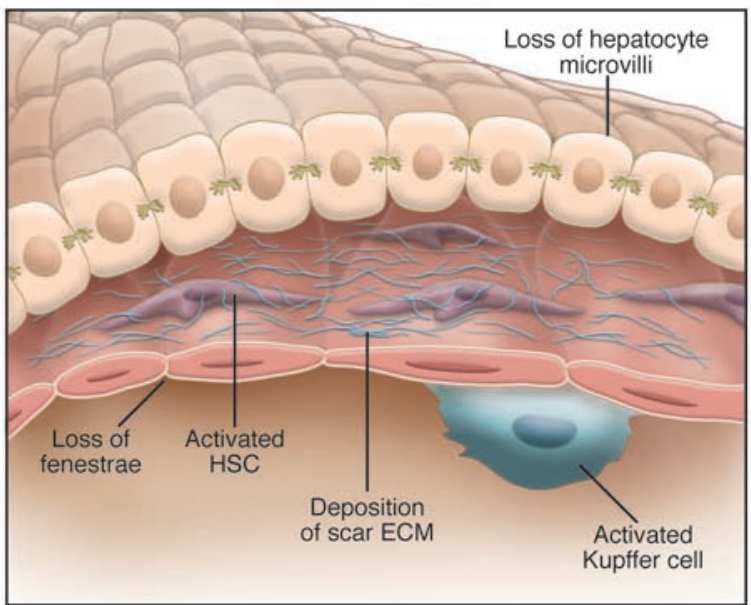

Figure 1

Sinusoidal events in the development of liver fibrosis. Injury to hepatocytes results in the recruitment and stimulation of inflammatory cells, as well as the stimulation of resident inflammatory cells (including Kupffer cells). Factors released by these inflammatory cells lead to transformation of HSCs into a myofibroblast-like phenotype. HSC activation leads to accumulation of scar (fibrillar) ECM. The presence of a fibrillar ECM in the Disse space has consequences for hepatocyte function, leading to the loss of microvilli and endothelial fenestrae. Therefore, the loss of normal tissue architecture contributes to impairment of organ function. Figure modified with permission from Journal of Biological Chemistry (119).

\section{Defining the functional attributes and the origin of the liver myofibroblast}

As in other tissues, the fibrotic component of the liver's woundhealing response is mediated by myofibroblasts. In the injured liver, the myofibroblast is potentially derived from a number of cellular sources, foremost of which is the hepatic stellate cell (HSC) (Figure 1) (9, 18-20). HSCs are perisinusoidal cells that normally reside in the Disse space and contain numerous retinoid and lipid droplets (9). During injury and in response to inflammatory and other stimuli, these cells gain a myofibroblast-like phenotype $(8,10,18,19)$ and represent a key effector cell in the fibrotic response (see below).

Historical perspective. Clearly the different model systems did not develop in a chronological order, although at one time or another each system has come to the fore to provide a platform of data from which a new area of research has developed. Histological studies have a history stretching back more than 100 years. Over this period of time, a detailed picture of the events that lead to the development of fibrosis and the histopathological characteristics of hepatic fibrosis have been catalogued in a detailed manner. Historical landmarks (reviewed in refs. 9 and 21) include the identification of the sternzellen by von Kupffer in 1876 and the first liver biopsy by Erlich and Lucatello in 1890. The sternzellen, which was subsequently called the Ito cell and is now known as the HSC, is now established as a major contributor to fibrotic processes in the liver (8-10) (Figure 1). More recently, the advent of effective antiviral regimens for the treatment of infection with either HBV or HCV have provided researchers with the opportunity to study the resolution of fibrosis that occurs when the insult that initiated the fibrosis is removed (7). Most recently, this data has been complemented with detailed studies of the pattern of hepatocyte loss, structural changes in the liver, and ECM turnover in examples of human cirrhosis (see below) (14).
The two most commonly used models of experimental fibrosis are iterative toxic damage (for example, elicited by $\mathrm{CCl}_{4}$ intoxication) and bile duct ligation (BDL) $(16,17)$. Other models mimicking specific disease attributes include special diets (for example, those that model non-alcoholic steatohepatitis [refs. 22-27]) and expression of hepatotropic viral proteins $(28,29)$.

Intoxication with $\mathrm{CCl}_{4}$ results in hepatocyte damage, necrosis, inflammation, and fibrosis, which spreads to link the vascular structures that feed into and drain the hepatic sinusoid (the portal tract and central vein radicle, respectively), and over 8-12 weeks results in the development of cirrhosis (16). BDL stimulates the proliferation of biliary epithelial cells and oval cells (which are hepatocyte progenitors), resulting in proliferating bile ductules with an accompanying portal inflammation and fibrosis $(17,30)$. Using the $\mathrm{CCl}_{4}$ intoxication model of liver fibrosis, in the 1970s, two groups established that fibrosis was associated with detectable collagenase activity, suggesting for the first time that progressive fibrosis might be characterized not only by changes in ECM synthesis but also by changes in the pattern of ECM degradation $(31,32)$. More recent studies have confirmed this observation and through a series of complementary approaches have, at least in part, characterized the mechanisms underlying the changes in the pattern of ECM degradation that accompany fibrosis and cirrhosis (33) (see below).

Many recent advances in the liver fibrosis field were underpinned by a series of pivotal studies using cell culture models of HSC activation to define HSC phenotype and behavior $(8,12,18,19)$. During the late 1980s, methods were established to isolate liver nonparenchymal cells in a highly reproducible manner. With the advent of methods to isolate HSCs, Kupffer cells, and endothelial cells reproducibly, it became clear that activated HSCs were the main source of hepatic collagens in fibrosis, and not hepatocytes as had hitherto been thought $(8,12,18)$. Indeed, it became clear that contaminating HSCs in hepatocyte cultures were the probable source of collagen secretion, which until that time had been 


\section{Table 1}

Representative examples of innate immune cell manipulation in animal models of liver fibrosis

$\begin{array}{ll}\text { Cell type } & \text { Method of inducing fibrosis } \\ \text { Macrophages } & \mathrm{CCl}_{4} \text { intoxication }\end{array}$

Cell type
Macrophages

$\mathrm{CCl}_{4}$ intoxication intoxication, or porcine serum in mice: bile duct resection or $\mathrm{CCl}_{4}$ intoxication

Neutrophils $\mathrm{BDL}$
Administration of $\alpha$-naphthylisothiocyanate
3,5-diethoxycarbonyl-1,4-
dihydrocollidine diet or
$\mathrm{CCl}_{4}$ intoxication

NK cells

\author{
Method of cell manipulation \\ Selective depletion of macrophages \\ during liver injury and repair
}

Mast cell-deficient mutant Ws/Ws rats and W/Wv mice

\author{
Effect on fibrosis \\ Functionally distinct subpopulations \\ of macrophages in the same tissue \\ have critical roles in both the \\ injury and recovery phases of \\ inflammatory scarring \\ No effect on development \\ of liver fibrosis
}

Refs.

62

In rats: administration of neutrophilspecific antiserum; in mice: transgenic expression of IL-8 causes an underlying defect in neutrophil function

Cxcr2--- mice with resultant $50 \%$ reduction in neutrophil recruitment Activation and depletion of NK cells
No effect on hepatic fibrogenesis

No effect on hepatic fibrosis

NK cells ameliorate liver fibrosis by killing activated HSCs

CXcr2---, CXC chemokine receptor 2-deficient.

erroneously ascribed to hepatocytes (18). This work was elegantly complemented by studies in which highly purified cell populations were isolated from experimentally injured liver, which confirmed that HSCs, and to a lesser extent endothelial cells, were the main source of collagen I in fibrotic injury in the liver (19).

HSCs as a source of wound-healing myofibroblasts. Current evidence suggests that the process of hepatic fibrosis is driven primarily by the development of inflammation in response to parenchymal injury. Indeed, evidence from both human studies and animal models demonstrates a correlation between inflammatory activity and fibrosis $(16,17,34-36)$. Furthermore, deletion of specific components of the inflammatory response modifies or attenuates the fibrotic response in vivo (see below and Tables 1 and 2). A characteristic feature of inflammation is the activation of HSCs to a myofibroblast-like phenotype, a phenotype that can be readily recapitulated in tissue culture.

Once isolated from normal liver, if quiescent HSCs are plated on uncoated tissue culture plastic in the presence of serum, they undergo a phenotypic change, which in many ways recapitulates that observed in vivo during experimental liver injury $(8,10)$. Over a period of five to ten days, the quiescent HSCs gain a myofibroblast-like phenotype - they express the intermediate filament $\alpha$-SMA, they proliferate, and they express profibrotic genes $(8,10,30)$. This model has proven a robust and reproducible one in which the cellular attributes of wound-healing myofibroblasts in the liver can be defined and manipulated; indeed, it has become apparent that activated HSCs have a wide repertoire of activities that emphasize the dynamic nature of the liver wound-healing response (Figure 2). These activities include the synthesis of fibrillar collagens, contractile activity, secretion of chemotactic and vasoactive factors, migratory activity, and the secretion of MMPs and tissue inhibitors of metalloproteinases (TIMPs) (7-9, 37-43).

\section{Regulation of HSC phenotype in liver injury}

In vivo there is a close topographical relationship between the site of inflammation and the development of fibrosis. Although there are a substantial number of leukocytes in the resting liver, liver injury results in a massive accumulation of recruited inflammatory cells, with contemporaneous activation of the resident inflammatory cells. There is evidence that inflammation promotes fibrosis through a number of mechanisms and cell mediators. Foremost among these is TGF- $\beta 1$, which has been identified as the most profibrotic cytokine, promoting HSC expression of collagen I, HSC transition to a myofibroblast-like phenotype, and HSC inhibition of ECM degradation through the expression of TIMPs (44). In parallel, PDGF has emerged as the most potent pro-proliferative cytokine for HSCs (45). For both of these cytokines, a mechanistic role for these mediators in the fibrotic process has been demonstrated in animal models $(46,47)$. Connective tissue growth factor (CTGF) $(48,49)$ has also recently emerged as a potential mediator of fibrogenesis.

The cell culture model of HSC activation has also facilitated the detailed study of nonsoluble regulatory factors present in the fibrotic microenvironment. Among these are cell-cell and cell-ECM interactions. The process of inflammation and repair results in substantial and potentially rapid changes in the ECM content adjacent to activated HSCs. In the normal liver, HSCs are close to a non-electron-dense basement membrane-like ECM (50). Following injury and fibrosis, this is degraded and replaced with an ECM rich in fibrillar collagens and other noncollagen ECM molecules and integrin ligands (50). An accumulating body of evidence now indicates that HSC-matrix interactions exert a profound influence on HSC behavior, regulating their activation, proliferation, survival, and cell cycle arrest (51-55). If culture-activated HSCs are replated on a basement membrane-like matrix, a reversal of activation is observed, and several of the markers of activation become downregulated, including expression of collagen and TIMPs $(51,54)$. Plating freshly isolated HSCs on a basement membrane-like matrix has the effect of preventing spontaneous activation. By contrast, the activated phenotype is promoted by plating quiescent HSCs on collagen I $(51,54)$. Other experiments blocking specific molecular HSC-ECM interactions have identified HSC expression of discoidin domain receptors and integrins as crucial for the effects of ECM on HSC behavior $(52,53,55)$. 


\section{Table 2}

Representative examples of adaptive immune cell manipulation in animal models of liver fibrosis

\section{Cell type Method of inducing fibrosis \\ T cells $\quad \mathrm{CCl}_{4}$ intoxication or thioacetamide}

$\mathrm{CCl}_{4}$ intoxication

Infection with Schistosoma mansoni

B cells $\quad \mathrm{CCl}_{4}$ intoxication

Infection with S. mansoni
Cell type affected or manipulated

Transgenic mice with hepatocyte expression of rat IL-10; adoptive transfer of various lymphocyte subsets to SCID mice

Series of $\mathrm{CCl}_{4}$-induced liver injury experiments with mice that lack CD4+ T cells, CD8 $+\mathrm{T}$ cells, $\gamma \delta$ $T$ cells, or both $B$ and $T$ cells Treatment of $S$. mansoni-infected mice with an inhibitor of IL-13

B cell-deficient mice

B cell-deficient mice

\section{Effect on fibrosis}

Fibrosis promoted by $\mathrm{CD} 8+\mathrm{T}$ cells in adoptive transfer experiment; $\mathrm{CD}^{+}{ }^{+} \mathrm{T}$ cell-mediated disease is attenuated by recombinant IL-10

$\mathrm{CD}^{+}, \mathrm{CD} 8^{+}$, and $\gamma \delta \mathrm{T}$ cells do not substantially affect hepatic fibrosis

Development of hepatic fibrosis blocked by IL-13 inhibitor during a Th2-dominated inflammatory response

Absence of $B$ cells attenuates liver fibrosis in an antibody- and T cell-independent manner Increased hepatic fibrosis
Refs.

74

75

76
An emerging area of interest is the role of ECM stiffness (as opposed to composition) as a mediator of HSC behavior. In a series of elegant studies, Wells and her group have developed culture models that demonstrate that HSC activation can be linked to the rigidity of the subcellular matrix (56). This mechanism of HSC activation might be particularly germane early in injury because tissue edema (which increases tissue rigidity) is a characteristic of inflammation.

Animal models have also proven to be highly effective in demonstrating the role of specific components of the inflammatory system in the development of fibrosis (for detailed review, see ref. 57). A brief summary of studies in which individual inflammatory cell types have been depleted, and the subsequent effect of this on the development of fibrosis, is given in Tables 1 and 2 . Macrophages have been demonstrated to promote fibrosis in a series of studies, are a potent source of the HSC activator TGF- $\beta 1$, and can regulate the HSC response to PDGF (58-62). NK cells might also profoundly affect the fibrotic response (63). However, other cells of the innate immune system that might be expected to provoke fibrosis, such as mast cells and neutrophils, seem to exert a less profound influence on experimental fibrosis (64-69). One of the main potential sources of PDGF in liver injury is, of course, the platelet. Remarkably, the role of platelets in liver fibrosis has not been studied in detail in vivo to date. Models of wound healing elsewhere in the body have successfully employed platelet depletion without dramatic results, but this work merits extension to models of liver injury $(70,71)$. The role of the clotting cascade has received much attention in studies of pulmonary fibrosis (72), and recent studies in liver fibrosis have implied a role, particularly for thrombin, in promoting the fibrotic response. Indeed, the procoagulant state associated with factor $\mathrm{V}$ Leiden is also associated with the progression of fibrosis in chronic HCV infection (73).

Animal models demonstrate that both $\mathrm{B}$ and $\mathrm{T}$ cells can regulate the fibrotic response in vivo $(74,75)$. Indeed, one of the most interesting observations to arise from studies of the differential susceptibility of mouse strains to fibrosis was that a Th2 cell response drives fibrosis more effectively than a Th1 cell response $(76,77)$. It is tempting to speculate that the response to liver infection with parasites, which is characterized as a Th 2 cell response, might have evolved to promote the development of an aggressive intrahepatic scarring process that in the short term might be expected to wall off and compartmentalize the parasite but that in the long term leads to progressive fibrosis $(76,77)$. Additionally, soluble factors have been shown to have a role in the pathogenesis of fibrosis in studies using gene knockout mice. These include adipokines, vasoactive substances, interleukins, and IFN- $\gamma$ (reviewed in ref. 5).

Tissue culture models have also demonstrated that HSCs can themselves regulate fibrosis and inflammation, both through autocrine expression of specific cytokines and through direct response to non-cytokine components of the inflamed microenvironment (78-80). The ingestion of apoptotic hepatocytes by HSCs, for example, leads to their increased secretion of TGF- $\beta 1$ in a manner analogous to that seen for macrophages $(78,79)$. HSCs also participate in the innate immune response directly, through expression of TLR4 (80). These data emphasize the dynamic role played by the activated HSC regulating the inflammatory and fibrotic responses in addition to mediating fibrogenesis.

Non-HSC origins of myofibroblasts in the fibrotic liver. Very recently, evidence has emerged from both animal models and human studies that liver myofibroblasts can be derived from BM stem cells (81-83). Additionally, there is evidence that periportal fibroblasts and myofibroblasts derived by epithelial-mesenchymal transition (EMT) might make up part of the fibrogenic cell population (see below) (Figure 3) (84-88).

Each portal tract contains a population of portal myofibroblasts that probably contribute to fibrotic diseases, such as viral hepatitis and autoimmune conditions, with a portal component. Intriguingly, comparative tissue culture studies suggest that activated HSCs proliferate more rapidly than portal myofibroblasts and might therefore represent the dominant resident liver myofibroblast cell population during fibrotic injury (89). Additionally it has been postulated that hepatic myofibroblasts might arise by EMT (88). It seems probable that, in the near future, sophisticated lineage tracking will be employed to define the contribution of EMT to liver fibrosis.

Recently there has been substantial interest in the role of stem cells in the pathogenesis of liver fibrosis. Although initiated by the observation in human liver that hepatocytes might be derived from BM (90-92), it seems increasingly probable that fusion of 


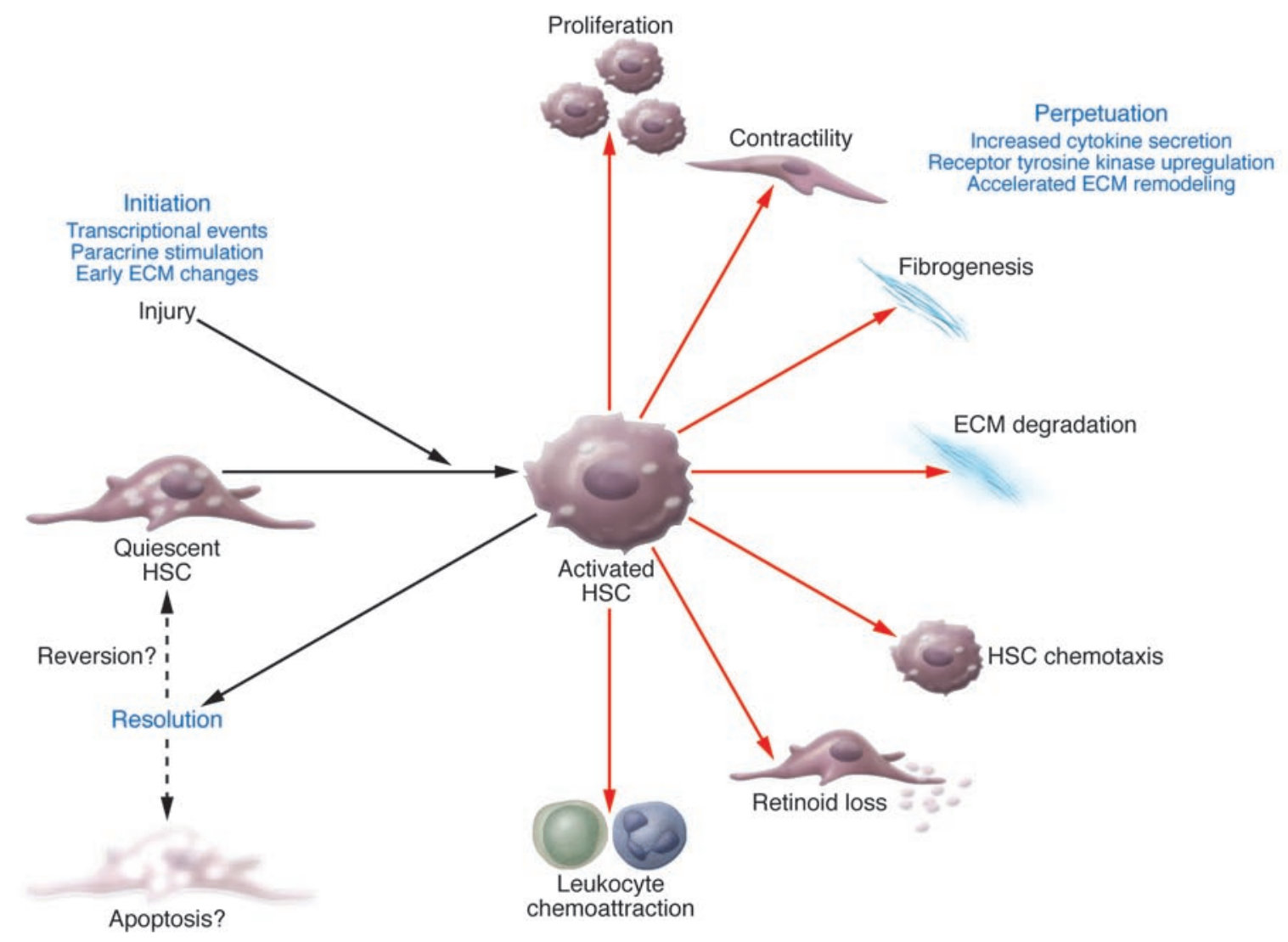

Figure 2

Repertoire of activities of the activated myofibroblast-like HSC. Activated HSCs, which are myofibroblast-like in phenotype, demonstrate a plastic and metabolically active phenotype that is proliferative, fibrogenic, and contractile. HSCs also release mediators that regulate ECM degradation (MMPs and TIMPs), chemotaxis, and leukocyte chemoattraction. The shedding of retinoids might be a critical, though as yet poorly defined, signal regulating fibrogenesis. During resolution of liver injury, activated HSCs and myofibroblasts have been demonstrated to undergo apoptosis. It is also possible that spontaneous reversion to a more quiescent phenotype might occur. Figure modified with permission from Journal of Biological Chemistry (119).

stem cells and hepatocytes accounts for this finding (90-92). Lineage tracking of cells in humans is impossible in most situations, although it has been possible in a series of male patients with sexmismatched liver transplants, who subsequently developed graft disease (i.e., fibrosis), and a single female patient who developed cirrhosis after receiving a BM transplant from a male (81). After Forbes and colleagues identified these individuals, they used $\mathrm{Y}$ chromosome tracking to identify the origin of the cells participating in liver fibrosis (81). Substantial numbers of scar-associated myofibroblasts in fibrotic areas were found to be BM derived (81). Subsequently, using a mouse $\mathrm{CCl}_{4}$-intoxication model of liver fibrosis in which sex-mismatched BM transplants were undertaken, the same group observed clear evidence of a BM contribution to the myofibroblasts within fibrotic scars (82). Additionally, there was evidence that the BM contributed to both the macrophage and HSC populations within the injured liver (82). This work was subsequently reproduced in a BDL model of liver fibrosis (83). Mouse models using sex-mismatched BM transplantation have dissected the bone marrow stem cell-liver axis in greater detail $(82,83)$. By subfractionating the BM stem cell compartment, it has been demonstrated that although hematopoietic stem cells contribute to the inflammatory cell infiltrate, the myofibroblast-like cells derived from the BM are of mesenchymal stem cell origin (82). Intriguingly, BM-derived cells are widely distributed within the scar in advanced fibrosis. That is, whatever the origin and topography of the injury in chronic disease, BM-derived myofibroblasts begin to replace local recruitment of myofibroblasts over time.

Evidence of a functional role for BM-derived myofibroblasts was provided by transplanting $\mathrm{BM}$ from genetically-modified mice into wild-type mice before inducing fibrosis with $\mathrm{CCl}_{4}(82)$. When BM was transplanted from mice bearing a reporter transgene for collagen, the recruited myofibroblasts were shown to transcribe this gene. Moreover, when wild-type mice were transplanted with BM from a transgenic mouse that develops a characteristic pattern of liver scarring because it expresses a form of collagen I not susceptible to degradation by MMPs, $\mathrm{CCl}_{4}$ administration induced the development of liver scarring with characteristics similar to those seen in the BM donor mouse (82). Therefore, transfer of genetically modified BM altered the phenotype of the liver fibrosis to reflect the genotype of the BM donor rather than the recipient mouse. Additionally, this study provided hard evidence that the recruited cells contribute directly to fibrosis through the expression, synthesis, and secretion of collagen I. These studies did not analyze the specific mechanisms by which the BM-derived cells are recruited 


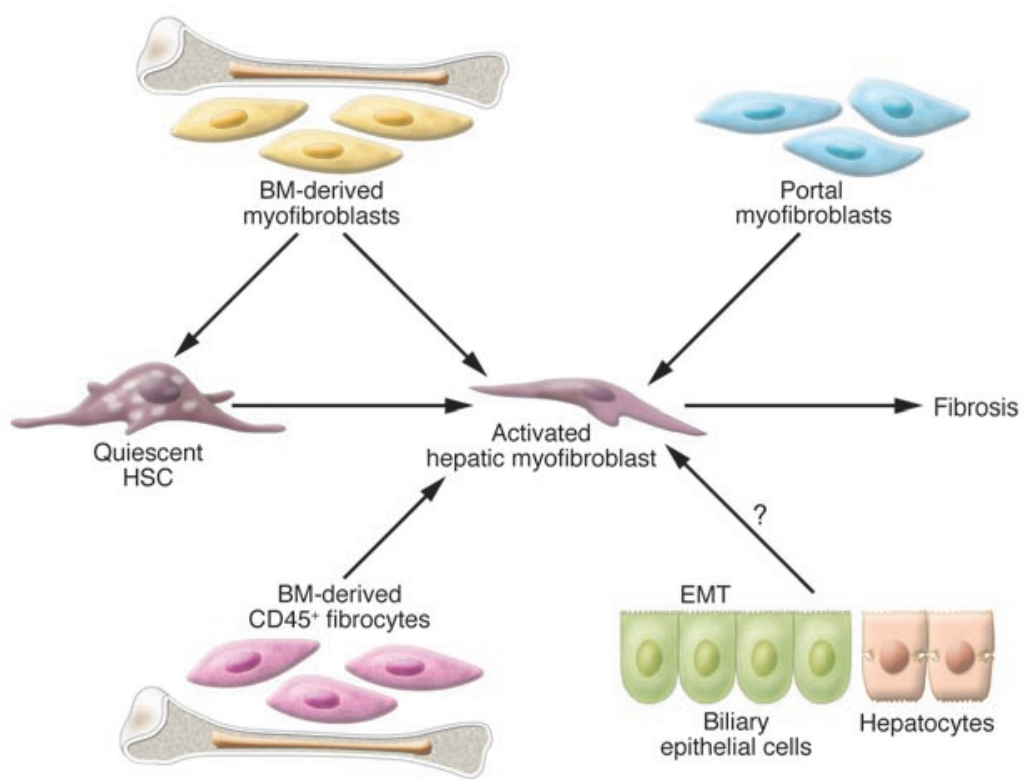

\section{Figure 3}

Diagramatic representation of the possible sources of liver myofibroblasts. There is considerable evidence supporting the notion that HSCs are a major source of myofibroblasts in the injured liver. Additionally, contributions to the myofibroblast population might come from portal myofibroblasts. Most recently, BM stem cells have been demonstrated to contribute to the inflammatory cell population and the myofibroblast population in the injured liver, which might occur directly or through an intermediary cell, such as a quiescent HSC or CD45+ fibrocyte. Research is currently underway to determine the role of EMT in the development of liver myofibroblasts. to the liver and did not identify whether the cells are recruited to directly become myofibroblasts or whether they require a transition through, say, an HSC phenotype. Some evidence from the BDL model, however, suggests that the cells might be recruited as $\mathrm{CD} 45^{+}$fibrocytes that enjoy a relatively widespread lymphoid organ distribution in injury but that transform to myofibroblasts in the liver in the presence of TGF- $\beta 1$ (83).

Plasticity of the liver myofibroblast. A further level of complexity in our understanding of the liver myofibroblast comes from recent studies aimed at monitoring the expression of characteristics perceived to define activated HSCs and myofibroblasts, namely, collagen I and $\alpha$-SMA. In a study using a dual reporter transgenic mouse in which expression of collagen I and $\alpha$-SMA could be detected independently, strong evidence emerged for functional differences between the periportal myofibroblasts (which were shown to express collagen I but not $\alpha$-SMA) and the myofibroblasts derived from HSCs (which were shown to express both collagen I and $\alpha$-SMA) (93). Moreover, after extracting HSCs from the liver of these transgenic mice, there was evidence in tissue culture for temporal changes in the expression pattern of individual genes, suggesting that there might be day-to-day variation in the expression of genes used to define the myofibroblast phenotype (93).

Taken together, these recent observations suggest that we need to be open minded about the origin of the fibrogenic cells in the liver, an origin that might change with the topography and duration of the pathology. Furthermore, perhaps rather than make assumptions about cell behavior and function on the basis of (oftentimes presumed) lineage, we should aim to define cells on the basis of function, in situ and in vivo.

ECM degradation. Although fibrosis was previously thought to be at best irreversible and at worst relentlessly progressive, data from animal models and human studies have recently challenged these ideas (7). Cell culture studies have provided clues to the mechanism underlying both ECM accumulation and degradation in liver fibrosis. As described above, the activation of HSCs and their transition to a myofibroblast-like phenotype is associated with increased expression of collagen I. The mechanisms and chronology of gene expression during activation are easily studied in tissue culture. For example, it has been demonstrated that in addition to enhanced transcription, changes in mRNA stability mediated by a $5^{\prime}$ loop in the mature transcript encoding collagen I are responsible for the increased amounts of mRNA encoding collagen I in activated HSCs (94). During activation in tissue culture, rodent HSCs demonstrate a distinct pattern of MMP expression. Early during the activation process, MMP-13 (also known as collagenase 3) and MMP-3 (also known as stromelysin) are transiently expressed $(30,95,96)$. As HSCs become more activated, expression of MMP-13 and MMP-3 decreases, whereas expression of MMP-2 (also known as gelatinase A), MMP-9 (also known as gelatinase B), and MMP-14 (also known as MT1-MMP) increases (97-101). Therefore, in terms of target substrates, HSCs undergoing activation express a true collagenase in combination with a promiscuous enzyme with degradative activity against several ECM components. By contrast, fully activated myofibroblast-like HSCs express MMPs that act against type IV collagen, the main component of the basement membrane-like matrix, degradation of which would be expected to further promote activation (see above). Expression of MMP-2 can also be important in mediating HSC proliferation, potentially by regulating ECM turnover $(54,99)$, and in combination with MMP-14 might confer degradative activity against collagen I (101). Studies of whole human and rat liver indicate that MMPs that act against several ECM components are expressed in end-stage cirrhosis (95, 97-102). However, during the process of HSC activation and before collagen I expression is increased, HSC expression of TIMP1 and TIMP2 is markedly increased $(30,99,103)$. Indeed, it is possible to demonstrate that the secreted MMPs, MMP-2 and MMP-9, are inhibited more than 20 -fold by HSC-derived TIMP1 (103). Subsequent studies using several animal models of progressive fibrosis and studies of explanted human liver have confirmed that fibrosis is characterized by an upregulation of TIMP1 and TIMP2 $(30,100)$.

These data led to the hypothesis that TIMPs regulate the pattern of ECM degradation that characterizes liver fibrosis by holding in check the activity of concurrently secreted MMPs (100-102). The implication of this observation is that liver fibrosis is potentially reversible and that ECM degradation should occur if the TIMP-MMP 
i.

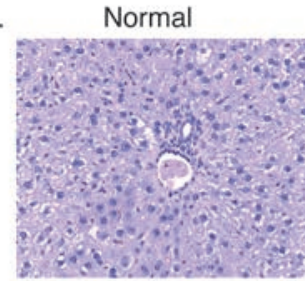

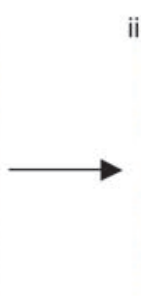

ii.

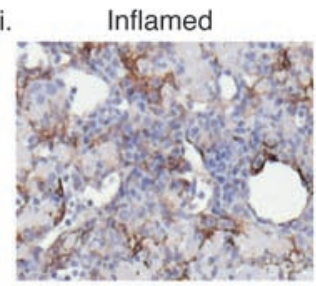

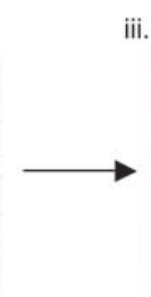

iii.

Fibrotic
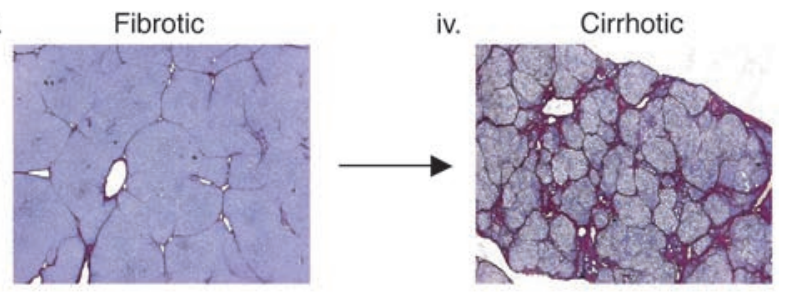

vi.

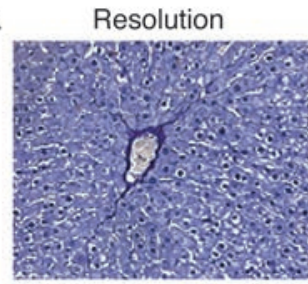

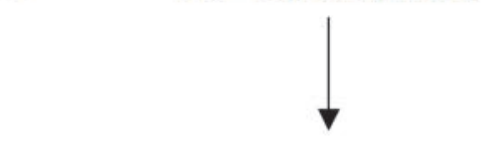

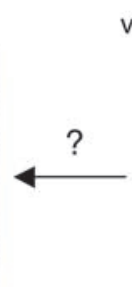

Remodeling of cirrhosis

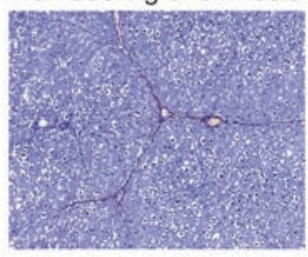

\section{Figure 4}

Liver cirrhosis is an example of dynamic wound healing. Damage to the normal liver (i) results in inflammation and activation of HSCs (ii; identified by immunohistochemistry, with staining for $\alpha$-SMA [brown]) to secrete fibrillar collagens, culminating in the development of fibrosis (iii) and ultimately cirrhosis (iv). Withdrawal of the injurious agent can allow remodeling of the fibrillar matrix, leading to attenuated cirrhosis (v). Spontaneous resolution of fibrosis after removal of injury results in a return to near-normal architecture (vi). Whether complete resolution of cirrhosis can occur is currently unknown. Figure modified with permission from BMJ (3).

balance is altered to favor ECM degradation. Studies of BDL- and $\mathrm{CCl}_{4}$-mediated liver fibrosis were undertaken to determine the reversibility of fibrosis and to confirm this prediction. Following withdrawal of $\mathrm{CCl}_{4}$ after four weeks of intoxication, an established fibrosis will undergo spontaneous resolution with remodeling of the ECM (104) (Figure 4). A return to a virtually normal liver architecture ensues. A similar sequence of events occurs in BDL-mediated fibrotic livers after bilio-jejunal anastamosis (105).

By studying multiple time points during the recovery process, it can be shown that the amounts of TIMP1 and TIMP2 decrease after the insult that induced fibrosis is withdrawn. In association with this decrease, hepatic collagenase activity increases and net ECM degradation occurs $(102,104)$. In parallel, the myofibroblasts are lost from the receding hepatic scar by apoptosis. The identification of myofibroblast apoptosis as a major feature of spontaneous resolution of fibrosis has led to substantial interest in how this process is regulated, with the aim of manipulating the hepatic scar (for detailed review, see ref. 106).

As one might expect from this data, subsequent mechanistic studies to modulate the TIMP-MMP balance have confirmed the powerful influence this ratio has on the development and resolution of fibrosis. Overexpression of TIMP1 in mice was not associated with fibrosis in the absence of injury. However, following $\mathrm{CCl}_{4}$ intoxication, TIMP1 overexpression enhanced fibrosis and prevented spontaneous resolution $(107,108)$. Adenovirus-mediated overexpression of MMP-8 (also known as neutrophil collagenase) in mice is associated with decreased fibrosis (109), and administration of neutralizing TIMP1-specific antibody decreases the collagen content in $\mathrm{CCl}_{4}$-induced fibrosis (110). A particularly ingenious approach to dysregulating the TIMP-MMP balance has been taken by Roeb and colleagues. They have engineered a nonfunctional form of MMP-9 that binds TIMPs and sequesters the MMP inhibitory activity $(111,112)$, thereby unharnessing the
ECM-degrading potential present in a tissue. This TIMP1-scavenging tool has been successfully deployed in $\mathrm{CCl}_{4}$-induced fibrosis to decrease collagen levels in the fibrotic tissue and to increase apoptosis of activated HSCs.

Taken together, these data indicate that during progressive fibrosis in wild-type animals, TIMPs are expressed at levels that are sufficiently high to prevent net ECM loss from the liver. Nevertheless, controlled ECM turnover might still occur, particularly at the cell surface, where the concentration of active MMPs is likely to be greatest. Furthermore, the degradative activity of activated HSCs is likely to be most effective against the normal basement membrane-like matrix, and as a result, any degradation will promote activation of HSCs, thus perpetuating the fibrotic response.

\section{ECM degradation in human liver disease}

Fortuitously, at the same time that the studies of the detailed chronology of spontaneous resolution in animal models emerged, the first large-scale trials of antiviral treatment for infection with either HBV or HCV began to be reported $(7,113,114)$. Reports of spontaneous resolution of liver fibrosis had hitherto been criticized for being anecdotal. However, the antiviral trials provided biopsy data recorded before and after attempted viral eradication, and for the first time, large-scale histological studies of the effect of withdrawal of a hepatic insult were available in a relevant human disease. These data confirmed that viral eradication was associated with a marked regression in fibrosis and provided evidence that human liver fibrosis was at least partially reversible. This phenomenon is also observed following withdrawal of other chronic hepatic insults (reviewed in refs. 7, 15, and 113-115).

Whether advanced cirrhosis undergoes remodeling to a normal, or near normal, liver architecture remains controversial. Cirrhosis is not simply extensive fibrosis. Rather it is an extensive fibrosis characterized by architectural disruption, aberrant hepatocyte 
regeneration, nodule formation, and vascular changes. Currently, although there is evidence for an improvement in cirrhosis following treatment of the underlying insult, it is not clear whether remodeling with restitution of normal architecture is possible, and this has recently been the subject of keen debate by experts in the field (116). There is some experimental evidence that, if sufficiently advanced, cirrhosis might reach a point of no return. Indeed, using the $\mathrm{CCl}_{4}$-intoxication model of liver fibrosis, we have demonstrated that even after a very protracted recovery period, the remodeling of advanced cirrhosis is limited and the liver remains cirrhotic (102) (Figure 4). However, the least mature ECM, which forms the micronodules, becomes degraded, leading to attenuated macronodular cirrhosis (102). An interesting observation is that the areas of fibrosis that do not undergo remodeling are extensively cross-linked and relatively rich in ECM molecules (such as elastin) that facilitate lysyl oxidase- and tissue TGase-mediated cross-linking (102), suggesting that ECM cross-linking might represent a point of no return in fibrosis. An additional feature of irreversible fibrosis is that the scars are relatively hypocellular, suggesting that incomplete ECM degradation occurs if the appropriate cellular mediators are absent (102). Although it seems premature to accept that resolution of cirrhosis to normal tissue architecture is possible, Wanless and colleagues have presented some striking data indicating that ECM remodeling occurs even in end-stage human liver disease (14). Together the data suggest that some areas of liver fibrosis are subject to and susceptible to ECM degradation, whereas others are not. The consequences of a failure of collagen I degradation during fibrosis have been defined using a mouse that expresses a collagen I molecule not susceptible to degradation by collagenolytic MMPs (117). In this model, spontaneous resolution of fibrosis does not occur and HSCs do not undergo apoptosis. Additionally, the hepatocyte proliferation that usually accompanies the resolution of fibrosis does not occur (117).

The identity and source of the key collagenase(s) mediating resolution of fibrosis is only now becoming established. Experimental depletion of macrophages at the onset of fibrosis resolution retards ECM degradation and the loss of activated HSCs (62). This suggests that macrophages might be essential for initiating
ECM degradation, potentially by expression of the interstitial collagenases MMP-1 (in humans), MMP-13 (in rodents), and MMP-8 (in humans and rodents). MMP-2 and MMP-14 are also expressed during recovery (in rodents); indeed, HSC apoptosis is associated with increased expression of MMP-2 (in humans) $(101,118)$.

\section{Conclusion}

The current hypothesis favored by my laboratory indicates that liver fibrosis is a highly dynamic pathological state with respect to myofibroblast lineages, phenotype, and behavior. Additionally, hepatic fibrosis is dynamic with respect to ECM modification, turnover, and degradation. This appreciation of the dynamic nature of liver wound healing and fibrosis has developed from the effective analysis of complementary cell culture, human disease, and experimental animal models and has stimulated researchers to identify new antifibrotic approaches. The development of an effective and targeted antifibrotic therapy in the near future is now a reality, although key challenges remain. These include defining the features of irreversible components of the fibrotic response, which might need a very specific therapeutic approach, and identifying methods of selectively targeting the liver with therapeutic biologicals. Finally, we need to identify a sensitive and robust method to monitor fibrosis noninvasively to minimize the requirement for liver biopsy as a means to gauge progression or regression of fibrosis in the context of future therapeutic trials.

\section{Acknowledgments}

J.P. Iredale acknowledges the advice of Stuart Forbes and Neil Henderson during preparation of this manuscript. He also gratefully acknowledges the support of the Medical Research Council United Kingdom, The Wellcome Trust, and the Children's Liver Disease Foundation for their support of his research activities.

Address correspondence to: John P. Iredale, Medical Research Council/University of Edinburgh Centre for Inflammation Research, Queen's Medical Research Institute, Edinburgh EH16 4TJ, United Kingdom. Phone: 44-131-242-6559; Fax: 44-131-242-6578; E-mail: John.Iredale@ed.ac.uk.
1. Friedman, S.L. 2003. Liver fibrosis - from bench to bedside. J. Hepatol. 38(Suppl. 1):S38-S53.

2. Gines, P., Cardenas, A., Arroyo, V., and Rodes, J. 2004. Management of cirrhosis and ascites. N. Engl. J. Med. 350:1646-1654.

3. Iredale, J.P. 2003. Cirrhosis: new research provides a basis for rational and targeted treatments. BMJ. 327:143-147.

4. Fallowfield, J.A., and Iredale, J.P. 2004. Targeted treatments for cirrhosis. Expert Opin. Ther. Targets. 8:423-435.

5. Bataller, R., and Brenner, D.A. 2005. Liver fibrosis. J. Clin. Invest. 115:209-231. doi:10.1172/ JCI200524282.

6. Albanis, E., and Friedman, S.L. 2001. Hepatic fibrosis. Pathogenesis and principles of therapy. Clin. Liver Dis. 5:315-334, v-vi.

7. Iredale, J.P. 2001. Hepatic stellate cell behaviour during resolution of liver fibrosis. Semin. Liver Dis. 21:427-436

8. Friedman, S.L., Roll, F.J., Boyles, J., and Bissell, D.M. 1985. Hepatic lipocytes: the principal collagen-producing cells of normal rat liver. Proc. Natl. Acad. Sci. U. S. A. 82:8681-8685.

9. Geerts, A. 2001. History, heterogeneity, developmental biology, and functions of quiescent hepatic stellate cells. Semin. Liver Dis. 21:311-335.

10. Friedman, S.L., et al. 1992. Isolated hepatic lipo- cytes and Kupffer cells from normal human liver: morphological and functional characteristics in primary culture. Hepatology. 15:234-243.

11. Pinzani, M., et al. 1992. Fat-storing cells as liverspecific pericytes. Spatial dynamics of agoniststimulated intracellular calcium transients. J. Clin. Invest. 90:642-646.

12. Otto, D.A., and Veech, R.L. 1980. Isolation of a lipocyte-rich fraction from rat liver nonparenchymal cells. Adv. Exp. Med. Biol. 132:509-517.

13. Benyon, R.C., Iredale, J.P., Goddard, S., Winwood, P.J., and Arthur, M.J.P. 1996. Increased expression of tissue inhibitor of metalloproteinases-1 and -2 relative to interstitial collagenase in fibrotic human liver. Gastroenterology. 110:821-831.

14. Wanless, I.R., Nakashima, E., and Sherman, M. 2000. Regression of human cirrhosis. Morphologic features and the genesis of incomplete septal cirrhosis. Arch. Pathol. Lab. Med. 124:1599-1607.

15. Dufour, J.F., DeLellis, R., and Kaplan, M.M. 1997. Reversibility of hepatic fibrosis is autoimmune hepatitis. Ann. Intern. Med. 127:981-985.

16. Constandinou, C., Henderson, N., and Iredale, J.P. 2005. Modelling liver fibrosis in rodents. Methods Mol. Med. 117:237-250.

17. Tsukamoto, H., Matsuoka, M., and French, S.W. 1990. Experimental models of hepatic fibrosis: a review. Semin. Liver Dis. 10:56-65.
18. Maher, J.J., Bissell, D.M., Friedman, S.L., and Roll, F.J. 1988. Collagen measured in primary cultures of normal rat hepatocytes derives from lipocytes within the monolayer. J. Clin. Invest. 82:450-459.

19. Maher, J.J., and McGuire, R.F. 1990. Extracellular matrix gene expression increases preferentially in rat lipocytes and sinusoidal endothelial cells during hepatic fibrosis in vivo. J. Clin. Invest. 86:1641-1648.

20. Gabele, E., Brenner, D.A., and Rippe, R.A. 2003. Liver fibrosis: signals leading to the amplification of the fibrogenic hepatic stellate cell. Front. Biosci. 8:D69-D77.

21. Franken, F.H., and Falk, H. 1999. History of hepatology. In Oxford textbook of clinical hepatology. 2nd edition. J. Bircher, J.-P. Benhamou, N. McIntyre, M. Rizetto, and J. Rodes, editors. Oxford University Press. Oxford, United Kingdom. 2067-2076.

22. Anstee, Q.M., and Goldin, R.D. 2006. Mouse models in non-alcoholic fatty liver disease and steatohepatitis research. Int. J. Exp. Pathol. 87:1-16.

23. Koteish, A., and Diehl, A.M. 2001. Animal models of steatosis. Semin. Liver Dis. 21:89-104.

24. Kirsch, R., et al. 2003. Rodent nutritional model of non-alcoholic steatohepatitis: species, strain and sex difference studies. J. Gastroenterol. Hepatol. 18:1272-1282.

25. Kulinski, A., Vance, D.E., and Vance, J.E. 2004. A choline-deficient diet in mice inhibits neither the 
CDP-choine pathway for phosphatidylcholine synthesis in hepatocytes nor apolipoprotein B secretion. J. Biol. Chem. 279:23916-23924.

26. Oliviera, C.P.M.S., et al. 2003. Vitamin C and vitamin $\mathrm{E}$ in prevention of nonalcoholic fatty liver disease (NAFLD) in choline deficient diet fed rats. Nutr. J. 2:9-14.

27. Raubenheimer, P.J., Nyirenda, M.J., and Walker, B.R. 2006. A choline deficient diet exacerbates fatty liver but attenuates insulin resistance and glucose intolerance in mice fed a high fat diet. Diabetes. 55:2015-2020.

28. Vig, P., et al. 2006. The sources of parenchymal regeneration after chronic hepatocellular liver injury in mice. Hepatology. 43:316-324.

29. Chisari, F.V., et al. 1985. A transgenic mouse model of the chronic hepatitis B surface antigen carrier state. Science. 230:1157-1160.

30. Iredale, J.P., et al. 1996. TIMP-1 and interstitial collagenase expression in liver fibrosis; implications for pathogenesis. Hepatology. 24:176-184.

31. Okazaki, I., and Maruyama, K. 1974. Collagenase activity in experimental hepatic fibrosis. Nature. 252:49-50.

32. Montfort, I., and Perez-Tamayo, R. 1978. Collagenase in experimental carbon tetrachloride cirrhosis of the liver. Am. J. Pathol. 92:411-420.

33. Arthur, M.J., Mann, D.A., and Iredale, J.P. 1998. Tissue inhibitors of metalloproteinases, hepatic stellate cells and liver fibrosis. J. Gastroenterol. Hepatol. 13(Suppl.):S33-S38.

34. Brunt, E.M. 2004. Nonalcoholic steatoherpatits. Semin. Liver Dis. 24:3-20.

35. Ludwig, J., Viggiano, T.R., McGill, D.B., and Oh, B.J. 1980. Nonalcoholic steatohepatitis: Mayo clinic experiences with a hitherto unnamed disease. Mayo Clin. Proc. 55:434-438.

36. Sorensen, T.I., et al. 1984. Prospective evaluation of alcohol abuse and alcoholic liver injury in men as predictors of development of cirrhosis. Lancet. 2:241-244.

37. Rockey, D.C. 2001. Hepatic blood flow regulation by stellate cells in normal and injured liver. Semin. Liver Dis. 21:337-349.

38. Milani, S., et al. 1990. Procollagen expression by nonparenchymal rat liver cells in experimental biliary fibrosis. Gastroenterology. 98:175-184.

39. Marra, F. 1999. Hepatic stellate cells and the regulation of liver inflammation. J. Hepatol. 31:1120-1130.

40. Olaso, E., et al. 2003. Proangiogenic role of tumoractivated hepatic stellate cells in experimental melanoma metastasis. Hepatology. 37:674-685

41. Friedman, S.L. 2000. Molecular regulation of hepatic fibrosis, an integrated cellular response to tissue injury. J. Biol. Chem. 275:2247-2250.

42. Bataller, R., et al. 2000. Angiotensin II induces contraction and proliferation of human hepatic stellate cells. Gastroenterology. 118:1149-1156.

43. Rockey, D.C. 2001. Cellular pathophysiology of portal hypertension and prospects for management with gene therapy. Clin. Liver Dis. 5:851-865.

44. Gressner, A.M., Weiskirchen, R., Breitkopf, K., and Dooley, S. 2002. Roles of TGF-beta in hepatic fibrosis. Front. Biosci. 7:d793-d807.

45. Pinzani, M., Gesualdo, L., Sabbah, G.M., and Abboud, H.E. 1989. Effects of platelet-derived growth factor and other polypeptide mitogens on DNA synthesis and growth of cultured rat liver fatstoring cells. J. Clin. Invest. 84:1786-1793.

46. George, J., Roulot, D., Koteliansky, V.E., and Bissell, D.M. 1999. In vivo inhibition of rat stellate cell activation by soluble transforming growth factor beta type II receptor: a potential new therapy for hepatic fibrosis. Proc. Natl. Acad. Sci. U. S. A 96:12719-12724

47. Borkham-Kamphorst, E., Stoll, D., Gressner, A.M., and Weiskirchen, R. 2004. Antisense strategy against PDGF B-chain proves effective in preventing experimental liver fibrogenesis. Biochem. Biophys. Res. Commun. 321:413-423.

48. Gao, R., and Brigstock, D.R. 2004. Connective tissue growth factor (CCN2) induces adhesion of rat activated hepatic stellate cells by binding of its C-terminal domain to integrin alpha(v)beta(3) and heparan sulfate proteoglycan. J. Biol. Chem. 279:8848-8855.

49. Williams, E.J., Gaca, M.D., Brigstock, D.R., Arthur, M.J., and Benyon, R.C. 2000. Increased expression of connective tissue growth factor in fibrotic human liver and in activated hepatic stellate cells. J. Hepatol. 32:754-761.

50. Schuppan, D., Ruehl, M., Sonasundaram, R., and Hahn, E.G. 2001. Matrix as a modulator of hepatic fibrogenesis. Semin. Liver Dis. 21:351-372.

51. Gaca, M.D., et al. 2003. Basement membrane-like matrix inhibits proliferation and collagen synthesis by activated rat hepatic stellate cells: evidence for matrix-dependent deactivation of stellate cells. Matrix Biol. 22:229-239.

52. Zhou, X., et al. 2004. Engagement of alphavbeta3 integrin regulates proliferation and apoptosis of hepatic stellate cells. J. Biol. Chem. 279:23996-24006.

53. Olaso, E., et al. 2001. DDR2 receptor promotes MMP-2-mediated proliferation and invasion by hepatic stellate cells. J. Clin. Invest. 108:1369-1378. doi:10.1172/JCI200112373.

54. Friedman, S.L., Roll, F.J., Boyles, J., Arenson, D.M., and Bissell, D.M. 1989. Maintenance of differentiated phenotype of cultured rat hepatic lipocytes by basement membrane matrix. J. Biol. Chem. 264:10756-10762.

55. Zhou, X., et al. 2006. Impaired proteolysis of collagen I inhibits proliferation of hepatic stellate cells: Implication for regulation of liver fibrosis. J. Biol. Chem. 281:39757-39765.

56. Wells, R.G. 2005. The role of matrix stiffness in hepatic stellate cell activation and liver fibrosis. J. Clin. Gastroenterol. 39(Suppl. 2):S158-S161.

57. Henderson, N.C., and Iredale,J.P. 2007. Liver fibrosis: cellular mechanisms of progression and resolution. Clin. Sci. 112:265-280

58. Matsuoka, M., and Tsukamoto, H. 1990. Stimulation of hepatic lipocyte collagen production by Kupffer cell-derived transforming growth factor beta: implication for a pathogenetic role in alcoholic liver fibrogenesis. Hepatology. 11:599-605.

59. Friedman, S.L., and Arthur, M.J. 1989. Activation of cultured rat hepatic lipocytes by Kupffer cell conditioned medium. Direct enhancement of matrix synthesis and stimulation of cell proliferation via induction of platelet-derived growth factor receptors. J. Clin. Invest. 84:1780-1785

60. Ide, M., Kuwamura, M., Kotani, T., Sawamoto, O, and Yamate, J. 2005. Effects of gadolinium chloride $(\mathrm{GdCl}(3))$ on the appearance of macrophage populations and fibrogenesis in thioacetamide-induced rat hepatic lesions. J. Comp. Pathol. 133:92-102.

61. Imamura, M., Ogawa, T., Sasaguri, Y., Chayama, K., and Ueno, H. 2005. Suppression of macrophage infiltration inhibits activation of hepatic stellate cells and liver fibrogenesis in rats. Gastroenterology. 128:138-146

62. Duffield, J.S., et al. 2005. Selective depletion of macrophages reveals distinct, opposing roles during liver injury and repair. J. Clin. Invest. 115:56-65. doi:10.1172/JCI200522675.

63. Radaeva, S., et al. 2006. Natural killer cells ameliorate liver fibrosis by killing activated stellate cells in NKG2D-dependent and tumor necrosis factor-related apoptosis-inducing ligand-dependent manners. Gastroenterology. 130:435-452.

64. Jeong, W.I., Lee, C.S., Park, S.J., Chung, J.Y., and Jeong, K.S. 2002. Kinetics of macrophages, myofibroblasts and mast cells in carbon tetrachloride-induced rat liver cirrhosis. Anticancer Res. 22:869-877.
65. Li, C.Y., and Baek, J.Y. 2002. Mastocytosis and fibrosis: role of cytokines. Int. Arch. Allergy Immunol. 127:123-126.

66. Sugihara, A., Tsujimura, T., Fujita, Y., Nakata, Y., and Terada, N. 1999. Evaluation of role of mast cells in the development of liver fibrosis using mast cell-deficient rats and mice. J. Hepatol. 30:859-867.

67. Okazaki, T., et al. 1998. Increase of mast cells in the liver and lung may be associated with but not a cause of fibrosis: demonstration using mast celldeficient Ws/Ws rats. Lab. Invest. 78:1431-1438.

68. Saito, J.M., Bostick, M.K., Campe, C.B., Xu, J., and Maher, J.J. 2003. Infiltrating neutrophils in bile duct-ligated livers do not promote hepatic fibrosis. Hepatol. Res. 25:180-191.

69. Xu, J., Lee, G., Wang, H., Vierling, J.M., and Maher, J.J. 2004. Limited role for CXC chemokines in the pathogenesis of alpha-naphthylisothiocyanateinduced liver injury. Am. J. Physiol. Gastrointest. Liver Physiol. 287:G734-G741.

70. Szpaderska, A.M., Egozi, E.I., Gamelli, R.L., and DiPietro, L.A. 2003. The effect of thrombocytopenia on dermal wound healing. J. Invest. Dermatol. 120:1130-1137.

71. Lesurtel, M., et al. 2006. Platelet-derived serotonin mediates liver regeneration. Science. 312:104-107.

72. Chambers, R.C., and Lurent, G.J. 2002. Coagulation cascade proteases and tissue fibrosis. Biochem. Soc. Trans. 30:194-200.

73. Wright, M., et al. 2003. Factor V Leiden polymorphism and the rate of fibrosis development in chronic hepatitis C virus infection. Gut. 52:1206-1210.

74. Safadi, R., et al. 2004. Immune stimulation of hepatic fibrogenesis by CD8 cells and attenuation by transgenic interleukin-10 from hepatocytes. Gastroenterology. 127:870-882.

75. Novobrantseva, T.I., et al. 2005. Attenuated liver fibrosis in the absence of B cells. J. Clin. Invest. 115:3072-3082. doi:10.1172/JCI24798.

76. Chiaramonte, M.G., Donaldson, D.D., Cheever, A.W., and Wynn, T.A. 1999. An IL-13 inhibitor blocks the development of hepatic fibrosis during a T-helper type 2-dominated inflammatory response. J. Clin. Invest. 104:777-785.

77. Shi, Z., Wakil, A.E., and Rockey, D.C. 1997. Strain-specific differences in mouse hepatic wound healing are mediated by divergent $\mathrm{T}$ helper cytokine responses. Proc. Natl. Acad. Sci. U. S. A. 94:10663-10668.

78. Canbay, A., et al. 2003. Apoptotic body engulfment by a human stellate cell line is profibrogenic. $L a b$. Invest. 83:655-663.

79. Fadok, V.A., et al. 1998. Macrophages that have ingested apoptotic cells in vitro inhibit proinflammatory cytokine production through autocrine/paracrine mechanisms involving TGF- $\beta$, PGE2, and PAF. J. Clin. Invest. 101:890-898.

80. Paik, Y.H., et al. 2003. Toll-like receptor 4 mediates inflammatory signaling by bacterial lipopolysaccharide in human hepatic stellate cells. Hepatology. 37:1043-1055

81. Forbes, S.J., et al. 2004. A significant proportion of myofibroblasts are of bone marrow origin in human liver fibrosis. Gastroenterology. 126:955-963.

82. Russo, F.P., et al. 2006. The bone marrow functionally contributes to liver fibrosis. Gastroenterology. 130:1807-1821.

83. Kisseleva, T., et al. 2006. Bone marrow-derived fibrocytes participate in pathogenesis of liver fibrosis. J. Hepatol. 45:429-438.

84. Kinnman, N., and Housset, C. 2002. Peribiliary myofibroblasts in biliary type liver fibrosis. Front. Biosci. 7:496-503.

85. Knittel, T., et al. 1999. Rat liver myofibroblasts and hepatic stellate cells: different cell populations of the fibroblast lineage with fibrogenic potential. Gastroenterology. 117:1205-1221.

86. Ramadori, G., and Saile, B. 2002. Mesenchymal cells in the liver-one cell type or two? Liver. 22:283-294. 
87. Cassiman, D., Libbrecht, L., Desmet, V., Denef, C., and Roskams, T. 2002. Hepatic stellate cell/myofibroblast subpopulations in fibrotic human and rat livers. J. Hepatol. 36:200-209.

88. Kalluri, R., and Neilson, E.G. 2003. Epithelialmesenchymal transition and its implications for fibrosis. J. Clin. Invest. 112:1776-1784. doi:10.1172/ JCI200320530.

89. Wells, R.G., Kruglov, E., and Dranoff, J.A. 2004. Autocrine release of TGF-beta by portal fiboblasts regulates cell growth. FEBS Lett. 559:107-110.

90. Alison, M.R., et al. 2000. Hepatocytes from nonhepatic adult stem cells. Nature. 406:257.

91. Alvarez-Dolado, M., et al. 2003. Fusion of bonemarrow-derived cells with Purkinje neurons, cardiomyocytes and hepatocytes. Nature. 425:968-973.

92. Alison, M.R., et al. 2004. Hepatic stem cells: from inside and outside the liver? Cell Prolif. 37:1-21.

93. Magness, S.T., Bataller, R., Yang, L., and Brenner, D.A. 2004. A dual reporter gene transgenic mouse demonstrates heterogeneity in hepatic fibrogenic cell populations. Hepatology. 40:1151-1159.

94. Stefanovic, B., Hellerbrand, C., and Brenner, A. 1999. Regulatory role of the conserved stem-loop structure at the $5^{\prime}$ end of collagen alpha1(I)mRNA. Mol. Cell. Biol. 19:4334-4342.

95. Benyon, R.C., and Arthur, M.J. 2001. Extracellular matrix degradation and the role of hepatic stellate cells. Semin. Liver Dis. 21:373-384.

96. Vyas, S.K., Leyland, H., Gentry, J., and Arthur, M.J. 1995. Rat hepatic lipocytes synthesize and secrete transin (stromelysin) in early primary culture. Gastroenterology. 109:889-898.

97. Arthur, M.J., Friedman, S.L., Roll, F.J., and Bissell, D.M. 1989. Lipocytes from normal rat liver release aneutral metalloproteinase that degrades basement membrane (type IV) collagen. J. Clin. Invest. 84:1076-1085.

98. Arthur, M.J., et al. 1992. Secretion of $72 \mathrm{kDa}$ type IV collagenase/gelatinase by cultured human lipocytes. Analysis of gene expression, protein synthesis and proteinase activity. Biochem. J. 287:701-707.

99. Benyon, R.C., Iredale, J.P., Goddard, S., Winwood, P.J., and Arthur, M.J. 1996. Expression of tissue inhibitor of metalloproteinases 1 and 2 is increased in fibrotic human liver. Gastroenterology. 110:821-831.

100.Benyon, R.C., et al. 1999. Progelatinase A is produced and activated by rat hepatic stellate cells and promotes their proliferation. Hepatology. 30:977-986.

101.Zhou, X., et al. 2004. Expression of matrix metalloproteinase- 2 and -14 persists during early resolution of experimental liver fibrosis and might contribute to fibrolysis. Liver Int. 24:492-501.

102.Issa, R., et al. 2004. Spontaneous recovery from micronodular cirrhosis: evidence for incomplete resolution associated with matrix cross-linking. Gastroenterology. 126:1795-1808.

103.Iredale, J.P., Murphy, G., Hembry, R.M., Friedman, S.L., and Arthur, M.J. 1992. Human hepatic lipcytes synthesize tissue inhibitor of metalloproteinases-1. Implications for regulation of matrix degradation in liver. J. Clin. Invest. 90:282-287.

104.Iredale, J.P., et al. 1998. Mechanisms of spontaneous resolution of rat liver fibrosis. Hepatic stellate cell apoptosis and reduced hepatic expression of metalloproteinase inhibitors. J. Clin. Invest. 102:538-549.

105.Issa, R., et al. 2001. Apoptosis of hepatic stellate cells: involvement in resolution of biliary fibrosis and regulation by soluble growth factors. Gut. 48:548-557.

106.Elsharkawy, A.M., Oakley, F., and Mann, D.A. 2005. The role and regulation of hepatic stellate cell apoptosis in reversal of liver fibrosis. Apoptosis. 10:927-939.

107.Yoshiji, H., et al. 2000. Tissue inhibitor of metalloproteinases-1 promotes liver fibrosis development in a transgenic mouse model. Hepatology. 32:1248-1254

108. Yoshiji, H., et al. 2002. Tissue inhibitor of metalloproteinases- 1 attenuates spontaneous liver fibrosis resolution in the transgenic mouse. Hepatology. 36:850-860.

109.Siller-Lopex, F., et al. 2004. Treatment with human metalloproteinase- 8 gene delivery ameliorates experimental rat liver cirrhosis. Gastroenterology.
126:1122-1133.

110.Parsons, C.J., et al. 2004. Antifibrotic effects of a tissue inhibitor of metalloproteinase- 1 antibody on established liver fibrosis in rats. Hepatology. 40:1106-1115.

111.Roeb, E., Behrmann, I., Grotzinger, J., Breuer, B., and Matern, S. 2000. An MMP-9 mutant without gelatinolytic activity as a novel TIMP-1 antagonist. FASEB J. 14:1671-1673.

112.Roderfeld, M., et al. 2006. Inhibition of hepatic fibrogenesis by matrix metalloproteinase- 9 mutants in mice. FASEB J. 20:444-454.

113. Dienstag, J.L., et al. 2003. Histological outcome during long-term lamivudine therapy. Gastroenterology. 124:105-117.

114.Poynard, T., et al. 2002. Impact of pegylated interferon alfa- $2 \mathrm{~b}$ and ribavirin on liver fibrosis in patients with chronic hepatitis C. Gastroenterology. 122:1303-1313

115. Hammel, P., et al. 2001. Regression of liver fibrosis after biliary drainage in patients with chronic pancreatitis and stenosis of the common bile duct. N. Engl. J. Med. 344:418-423.

116. Desmet, V.J., and Roskams, T. 2004. Cirrhosis reversal: a duel between dogma and myth. J. Hepatol. 40:860-867.

117. Issa, R., et al. 2003. Mutation in collagen-1 that confers resistance to the action of collagenase results in failure of recovery from CC14-induced liver fibrosis, persistance of activated hepatic stellate cells and diminished hepatocyte regeneration. FASEB J. 17:47-49.

118.Preaux, A.M., D’ortho, M.P., Bralet, M.P., Laperche, Y., and Mavier, P. 2002. Apoptosis of human hepatic myofibroblasts promotes activation of matrix metalloproteinase-2. Hepatology. 36:615-622.

119.Friedman, S.L. 2000. Molecular regulation of hepatic fibrosis, an integrated cellular response to tissue injury. J. Biol. Chem. 275:2247-2250.

120.Ferru, I., Roye, O., Delacre, M., Auriault, C., and Wolowczuk, I. 1998. Infection of B-cell-deficient mice by the parasite Schistosoma mansoni: dominstration of the participation of B cells in granuloma modulation. Scand. J. Immunol. 48:233-240. 